\title{
A DIMENSION FORMULA FOR HERMITIAN MODULAR CUSP FORMS OF DEGREE TWO ${ }^{1}$
}

\author{
MINKING EIE
}

\begin{abstract}
An explicit dimension formula for the vector space of Hermitian modular cusp forms of degree two with respect to the modular group $\Gamma_{2}(Z[i])=\operatorname{SU}(2,2) \cap$ $M_{4}(\mathbf{Z}[i])$ is obtained via the Selberg trace formula and its arithmetic properties. Also, a generating function for the graded ring of Hermitian cusp forms of degree two is given.
\end{abstract}

Introduction and notations. Denote by $E_{n}$ the unit matrix and by 0 the zero matrix in the matrix ring $M_{n}(\mathbf{C})$. Put

$$
J=\left[\begin{array}{cc}
0 & E_{n} \\
-E_{n} & 0
\end{array}\right] .
$$

The Hermitian symplectic group of degree $n, \Omega_{n}$, is then defined as the subgroup of matrices satisfying ${ }^{\mathrm{t}} \bar{M} J M=J$ in $M_{2 n}(\mathrm{C})$; i.e.

$$
\Omega_{n}=\left\{\left.M \in M_{2 n}(\mathbf{C})\right|^{\mathrm{t}} \bar{M} J M=J\right\} .
$$

Here ${ }^{\mathrm{t}} \bar{M}$ is the transpose complex conjugate to $M$.

Let $H_{n}$ be the Hermitian upper half-plane; specifically,

$$
H_{n}=\left\{Z \in M_{n}(\mathbf{C}) \mid Z=X+i Y, X={ }^{t} \bar{X}, Y={ }^{t} \bar{Y}>0\right\} .
$$

The Hermitian symplectic group $\Omega_{n}$ operators on $H_{n}$ transitively by the action

$$
M: Z \rightarrow M(Z)=(A Z+B)(C Z+D)^{-1}, \quad M=\left[\begin{array}{ll}
A & B \\
C & D
\end{array}\right] \in \Omega_{n} .
$$

For a given imaginary quadratic number field $\mathbf{F}$, we denote by $\mathbf{K}$ its ring of algebraic integers. The Hermitian modular group of degree $n, \Gamma_{n}(\mathbf{K})$, is defined as

$$
\Gamma_{n}(\mathbf{K})=\Omega_{n} \cap M_{2 n}(\mathbf{K}) .
$$

Let $S\left(k ; \Gamma_{n}(\mathbf{K})\right)$ be the space of holomorphic function $f(Z)$ on $H_{n}$ with $f(Z)$ satisfying the following conditions:

(1) $f(M(Z))=\operatorname{det}(C Z+D)^{k} f(Z)$ for all $M=\left[\begin{array}{cc}{ }_{C}^{A} & B \\ D\end{array}\right]$ in $\Gamma_{n}(\mathbf{K}), Z \in H_{n}$,

(2) $(\operatorname{det} Y)^{k / 2} f(Z)$ is bounded on $H_{n}, Z=X+i Y$.

A function in $S\left(k ; \Gamma_{n}(\mathbf{K})\right)$ is called a Hermitian modular cusp form of weight $k$ and degree $n$ with respect to $\Gamma_{n}(\mathbf{K})$. For a nonnegative integer $k$, it is well known that $S\left(k ; \Gamma_{n}(\mathbf{K})\right)$ is a finite dimensional Hilbert space [9 or 10]. Furthermore, its

Received by the editors June 24, 1985.

1980 Mathematics Subject Classification. Primary 10D20.

${ }^{1}$ This work was supported by Academia Sinica in Taiwan and Mathematisches Institut der Universität, Göttingen. 
dimension can be written as an integral of a Bergman kernel function of certain Hilbert space over a fundamental domain on $H_{n}$ with respect to $\Gamma_{n}(\mathbf{K})$ when $k$ is sufficiently large. This is called the Selberg trace formula.

More precisely, let

with

$$
K\left(Z_{1}, Z_{2}\right)=C(k, n)\left[\operatorname{det}\left(\frac{1}{2 i}\left(Z_{1}-{ }^{t} \bar{Z}_{2}\right)\right)\right]^{-k}
$$

$$
C(k, n)=2^{-n^{2}-n} \pi^{-n^{2}} \prod_{0 \leqslant i, j \leqslant n-1}(k-2 n+1+i+j) .
$$

Then for $k>(4 n-2)$, we have [7 or 9 ]

$$
\operatorname{dim}_{\mathbf{C}} S\left(k ; \Gamma_{n}(\mathbf{K})\right)=\int_{F_{n}} \sum_{\gamma \in \overline{\Gamma_{n}(\mathbf{K})}} K(Z, \gamma(Z)) \overline{j(\gamma, Z)}^{-k}(\operatorname{det} Y)^{k-2 n} d Z,
$$

where

(1) $j(\gamma, Z)=\operatorname{det}(C Z+D)$ if $\gamma=\left[\begin{array}{ll}A & B \\ C & D\end{array}\right] \in \Gamma_{n}(\mathbf{K})$,

(2) $\overline{\Gamma_{n}(\mathbf{K})}$ is the quotient group $\Gamma_{n}(\mathbf{K}) / U$ with $U$ the center of $\Gamma_{n}(\mathbf{K})$,

(3) $F_{n}$ is a fundamental domain on $H_{n}$ with respect to $\Gamma_{n}(\mathbf{K})$,

(4) $Z=X+i Y$ in $H_{n}$ and $d Z=d X d Y$ is the Euclidean measure on $C^{n^{2}}$.

In this paper, we shall consider the case $n=2, \mathbf{F}=\mathbf{Q}[i]$ and $\mathbf{K}=\mathbf{Z}[i]$. Finally, we get the following theorem.

MaIN TheOREM. Suppose $k$ is an even integer greater than 6. Then the dimension formula of the vector space of Hermitian modular cups forms of degree 2 and weight $k$ with respect to $\Gamma_{2}(\mathbf{Z}[i])$ is given by

$$
\begin{aligned}
\operatorname{dim}_{\mathbf{C}} S\left(k ; \Gamma_{2}(\mathbf{Z}[i])\right)= & 2^{-10} 3^{-3} 5^{-1}(k-1)(k-2)^{2}(k-3) \\
& +2^{-10} 3^{-3}(k-2)^{2}[581,29 ; 4] \\
& -2^{-3} 3^{-3}(k-2)[16,9,20,13,18,5 ; 12] \\
& +2^{-6} 3^{-2}[-249,0,-57,128,423,-64 ; 12] \\
& +5^{-1}[1,0,1,0,0 ; 10] .
\end{aligned}
$$

Here $\alpha(k)=\left[a_{0}, a_{1}, \ldots, a_{m-1} ; 2 m\right]$ means $\alpha(k)=a_{j}$ if $k \equiv 2 j(\bmod 2 m), 0 \leqslant j \leqslant$ $m-1$.

With a certain modification [5 or $\mathbf{1 2}$ or 15], we can exchange the summation and integral in the Selberg trace formula so that we can compute the contribution from a certain conjugacy class or a family of conjugacy classes, as has already been done in the real case [ 5 or 12 or 15]. Of course, the contribution from certain conjugacy classes which are zero is already known [15].

After conjugacy classes with zero contribution are excluded, it now remains to compute the contributions from those conjugacy classes with characteristic polynomials which are products of cyclotomic polynomials and their divisors over $\mathbf{Z}[i]$. Thus we can write the dimension formula as a finite sum of products of certain zeta functions and integrals. All these integrals can be evaluated by [7] for regular elliptic cases or by a direct calculation except for the contribution from the identity. Meanwhile, zeta functions involved in the formula can be evaluated except for a zeta 
function associated with the vector space of $2 \times 2$ Hermitian matrices studied by Sintani [16]. The contribution from the identity can be determined by certain arithmetic properties and an estimation of the volume of a fundamental domain. Thus we get a dimension formula with an undetermined constant which can be determined by the fact $\operatorname{dim}_{\mathbf{C}} S\left(8 ; \Gamma_{2}(\mathbf{Z}[i])\right)=1$ or $\operatorname{dim}_{\mathbf{C}} S\left(10 ; \Gamma_{2}(\mathbf{Z}[i])\right)=0$ [9]. Finally, we get that

$$
\sum_{k=8}^{\infty} \operatorname{dim}_{\mathbf{C}} S\left(k ; \Gamma_{2}(\mathbf{Z}[i])\right)=\frac{P(T)}{\left(1-T^{4}\right)\left(1-T^{10}\right)\left(1-T^{12}\right)^{2}\left(1-T^{16}\right)}
$$

with

$$
\begin{aligned}
P(T)= & T^{8}+2 T^{16}-T^{18}+2 T^{20}+T^{22}+2 T^{24}-T^{26}+T^{28}-2 T^{32}-T^{36} \\
& -2 T^{40}+2 T^{42}+T^{46}+T^{48}+2 T^{50} .
\end{aligned}
$$

1. Conjugacy classes in $\Gamma_{2}(Z[i])$. Classification of conjugacy classes in $\Gamma_{2}(\mathbf{Z}[i])$ under the conjugation of $\Omega_{2}=\mathrm{SU}(2,2)$ can be done as in the real case, for example, see [15]. Here we are only interested in those elements with characteristic polynomials which are products of cyclotomic polynomials and their divisors over $\mathbf{Z}[i]$.

Lemma 1. Suppose $M \in \Gamma_{2}(Z[i])$ and suppose it is possible for the contribution of the conjugacy classes represented by $M$ to be nonzero. Then the characteristic polynomial of $M$ is a product of the following polynomials:

$$
\begin{aligned}
& X-1, X+1, X-i, X+i, X^{2}+1, X^{2}+X+1, X^{2}-X+1, X^{2}+i X-1, \\
& X^{2}-i X-1, X^{2}-i, X^{2}+i, X^{4}+1, X^{4}+X^{3}+X^{2}+X+1, \\
& X^{4}-X^{3}+X^{2}-X+1, X^{4}-i X^{2}-1, X^{4}+i X^{2}-1 .
\end{aligned}
$$

Lemma 2. Suppose $M \in \Gamma_{2}(Z[i])$ and possesses the property in Lemma 1 . Then $M$ is conjugate in $\mathrm{SU}(2,2)$ to one of the following:

$$
\begin{aligned}
& {[S, U] ; \quad U=E_{2}, \quad\left[\begin{array}{rr}
1 & 0 \\
0 & -1
\end{array}\right],\left[\begin{array}{rr}
0 & 1 \\
-1 & 0
\end{array}\right],\left[\begin{array}{rr}
1 & -1 \\
1 & 0
\end{array}\right],\left[\begin{array}{ll}
0 & 1 \\
i & 0
\end{array}\right],\left[\begin{array}{ll}
1 & 0 \\
0 & i
\end{array}\right] ;} \\
& {\left[\begin{array}{rr}
\cos \theta & \sin \theta \\
-\sin \theta & \cos \theta
\end{array}\right] \times\left[\begin{array}{cc}
u & s u \\
0 & u
\end{array}\right], \quad u=1,-1, \text { i or }-i} \\
& {\left[\begin{array}{rrrr}
0 & 0 & 1 & * \\
0 & i & * & * \\
-1 & 0 & 0 & 0 \\
0 & 0 & 0 & i
\end{array}\right] \in \mathrm{SU}(2,2) ;} \\
& e\left[\frac{1}{2}, \frac{1}{2}, \frac{3}{2}, \frac{3}{2}\right], \quad e\left[\frac{1}{4}, \frac{5}{4}, \frac{7}{4}, \frac{3}{4}\right], \quad e\left[\frac{3}{4}, \frac{7}{4}, \frac{5}{4}, \frac{1}{4}\right], \\
& e\left[\frac{1}{4}, \frac{3}{4}, \frac{7}{4}, \frac{5}{4}\right], \quad e\left[\frac{1}{3}, \frac{1}{3}, \frac{5}{3}, \frac{5}{3}\right], \quad e\left[\frac{2}{3}, \frac{2}{3}, \frac{4}{3}, \frac{4}{3}\right], \\
& e\left[\frac{1}{3}, \frac{2}{3}, \frac{5}{3}, \frac{4}{3}\right], \quad e\left[\frac{1}{3}, \frac{4}{3}, \frac{5}{3}, \frac{2}{3}\right], \quad e\left[\frac{2}{3}, \frac{5}{3}, \frac{4}{3}, \frac{1}{3}\right], \\
& e\left[\frac{1}{6}, \frac{7}{6}, \frac{11}{6}, \frac{5}{6}\right], \quad e\left[\frac{5}{6}, \frac{11}{6}, \frac{7}{6}, \frac{1}{6}\right], \quad e\left[\frac{1}{2}, \frac{1}{3}, \frac{3}{2}, \frac{5}{3}\right], \\
& \text { (4) } e\left[\frac{1}{2}, \frac{2}{3}, \frac{3}{2}, \frac{4}{3}\right], \quad e\left[\frac{1}{2}, \frac{4}{3}, \frac{3}{2}, \frac{2}{3}\right], \quad e\left[\frac{1}{2}, \frac{5}{3}, \frac{3}{2}, \frac{1}{3}\right] \text {, } \\
& e\left[\frac{2}{5}, \frac{4}{5}, \frac{8}{5}, \frac{6}{5}\right], \quad e\left[\frac{4}{5}, \frac{8}{5}, \frac{6}{5}, \frac{2}{5}\right], \quad e\left[\frac{6}{5}, \frac{2}{5}, \frac{4}{5}, \frac{8}{5}\right], \\
& e\left[\frac{8}{5}, \frac{6}{5}, \frac{2}{5}, \frac{4}{5}\right], \quad e\left[\frac{1}{3}, \frac{5}{6}, \frac{5}{3}, \frac{1}{6}\right], \quad e\left[\frac{2}{3}, \frac{1}{6}, \frac{4}{3}, \frac{5}{6}\right], \\
& e\left[\frac{1}{2}, \frac{5}{6}, \frac{3}{2}, \frac{1}{6}\right], \quad e\left[\frac{1}{2}, \frac{1}{6}, \frac{3}{2}, \frac{5}{6}\right], \quad e\left[\frac{1}{12}, \frac{5}{12}, \frac{17}{12}, \frac{13}{12}\right], \\
& e\left[\frac{4}{3}, \frac{1}{6}, \frac{2}{3}, \frac{5}{6}\right], \quad e\left[\frac{5}{3}, \frac{5}{6}, \frac{1}{3}, \frac{1}{6}\right] \text {. }
\end{aligned}
$$


Here we use the following notations, also used in [5].

$$
\begin{gathered}
{[S, U]=\left[\begin{array}{ll}
E & S \\
0 & E
\end{array}\right]\left[\begin{array}{cc}
U & 0 \\
0 & \multicolumn{1}{c}{\bar{U}^{-1}}
\end{array}\right],} \\
{\left[\begin{array}{ll}
a & b \\
c & d
\end{array}\right] \times\left[\begin{array}{ll}
p & q \\
r & s
\end{array}\right]=\left[\begin{array}{cccc}
a & 0 & b & 0 \\
0 & p & 0 & q \\
c & 0 & d & 0 \\
0 & r & 0 & s
\end{array}\right],} \\
e[a, b, c, d]=\operatorname{diag}\left[e^{\pi i a}, e^{\pi i b}, e^{\pi i c}, e^{\pi i d}\right] .
\end{gathered}
$$

Proofs of Lemmas 1 and 2 are elementary, so we omit them here.

The Satake compactification of $\Gamma_{2}(\mathbf{Z}[i]) \backslash H_{2}$ is given by [9]

$$
X_{2}=\Gamma_{2}(\mathbf{Z}[i]) \backslash H_{2} \cup \Gamma_{1} \backslash H_{1} \cup\{i \infty\} .
$$

Here $H_{1}$ is the upper half-palne and $\Gamma_{1}=\mathrm{SL}_{2}(\mathbf{Z})$. Elements in Lemma 2 (1) are in the stabilizer of the zero dimensional cusp $\{i \infty\}$ and elements in Lemma 2 (2) and (3) are in the stabilizer of the one dimensional cusp $\Gamma_{1} \backslash H_{1}$. Elements in (4) are representatives of regular elliptic elements in $\Gamma_{2}(\mathbf{Z}[i])$; i.e. they have isolated fixed points in $\mathrm{H}_{2}$.

Let $\Gamma_{2}^{1}$ and $\Gamma_{2}^{0}$ be stabilizers of $\Gamma_{2}(\mathbf{Z}[i])$ at a one dimensional cusp and a zero dimensional cusp, respectively. Precisely, we let

$$
\begin{aligned}
& \Gamma_{2}^{1}=\left\{M \in \Gamma_{2}(\mathbf{Z}[i]) \mid M=\left[\begin{array}{llll}
a & 0 & b & * \\
* & u & * & * \\
c & 0 & d & * \\
0 & 0 & 0 & u
\end{array}\right]\right\}, \\
& \Gamma_{2}^{0}=\left\{M \in \Gamma_{2}(\mathbf{Z}[i]) \mid M=[S, U]\right\} .
\end{aligned}
$$

By the reduction theory of symplectic groups $[3$ or $\mathbf{8}]$ and keeping in mind that the Satake compactification $X_{2}$ of $\Gamma_{2}(\mathbf{Z}[i]) \backslash H_{2}$ has only two cusps up to $\Gamma_{2}(\mathbf{Z}[i])$ equivalence, we then have the following.

Proposition 1. Suppose $M \in \Gamma(\mathbf{Z}[i])$ and the contribution of the conjugacy classes represented by $M$ has the possibility of being nonzero. Then $M$ is conjugate in $\Gamma_{2}(\mathbf{Z}[i])$ to an element of $\Gamma_{2}^{1} \cup \Gamma_{2}^{0}$ or $M$ is conjugate in $\mathrm{SU}(2,2)$ to an element as in Lemma 2 (4).

In the remaining sections we compute contributions from conjugacy classes of regular elliptic elements, conjugacy classes in $\Gamma_{2}^{1}$ and $\Gamma_{2}^{0}$.

2. Contributions from conjugacy classes of regular elliptic elements. To compute the total contribution from conjugacy classes of regular elliptic elements, it is unnecessary to classify their conjugacy classes explicitly [12]. The total contribution can be written as $\Sigma_{\gamma} M(\gamma) \cdot I(\gamma)$ where $\gamma$ ranges over all representatives of con- 
jugacy classes of regular elliptic elements as in Lemma 2 (4). Here

$$
M(\gamma)=\sum \frac{1}{\left|C\left(\gamma^{\prime}, \Gamma_{2}(\mathbf{Z}[i])\right)\right|}
$$

where $\gamma^{\prime}$ ranges over all representatives of conjugacy classes which are conjugate in $\mathrm{SU}(2,2)$ to $\gamma$ and $\left|C\left(\gamma^{\prime}, \Gamma_{2}(\mathbf{Z}[i])\right)\right|$ is the order of centralizer $C\left(\gamma^{\prime}, \Gamma_{2}(\mathbf{Z}[i])\right)$ of $\gamma^{\prime}$ in $\Gamma_{2}(\mathbf{Z}[i])$. For each fixed $\gamma, M(\gamma)$ is a kind of Siegel mass that can be evaluated explicitly by the method of [12].

The integral $I(\lambda)$ has already been evaluated in [7]. If $\gamma$ is conjugate in $\operatorname{SU}(2,2)$ to $\operatorname{diag}\left[\lambda_{1}, \lambda_{2}, \lambda_{3}, \lambda_{4}\right]$, then we have

$$
I(\gamma)=\left(\lambda_{3} \lambda_{4}\right)^{k} /\left(1-\bar{\lambda}_{1} \lambda_{3}\right)\left(1-\bar{\lambda}_{1} \lambda_{4}\right)\left(1-\bar{\lambda}_{2} \lambda_{3}\right)\left(1-\bar{\lambda}_{2} \lambda_{4}\right) .
$$

Now by the representatives of conjugacy classes in Lemma 2 (4) and the same method employed in [12], we get

Proposition 2. The total contribution from conjugacy classes of regular elliptic elements in $\Gamma_{2}(\mathbf{Z}[i])$ to the dimension formula is given by

$$
\begin{aligned}
& \alpha+\beta \times[1,-1 ; 4]+29 \cdot 2^{-3} 3^{-3} \times[-1,-2,-1,1,2,1 ; 12] \\
+ & 79 \cdot 2^{-3} 3^{-4} \times[-1,2,-1 ; 6]+5^{-2} \times[3,-2,3,-2,-2 ; 10] .
\end{aligned}
$$

Here $\alpha$ and $\beta$ are constants independent of $k$.

REMARK. We need not have spent time determining $\alpha$ and $\beta$ since the same kind of unknown constants will occur in our calculation. So we can wait until the last stage to combine them together and determine their sum.

3. Contributions from conjugacy class in $\Gamma_{2}^{1}$. Conjugacy classes of $\Gamma_{2}^{1}$ can be classified by the elementary method employed in the real case, so we do not repeat it here. Besides those conjugacy classes in $\operatorname{Sp}(2, \mathbf{Z})$ listed in [11], we have the following new conjugacy classes for $\Gamma_{2}^{1}$ :

$$
\begin{gathered}
{\left[\begin{array}{rr}
0 & 1 \\
-1 & 1
\end{array}\right] \times\left[\begin{array}{ll}
u & s u \\
0 & u
\end{array}\right], \quad s \in \mathbf{Z}, u=i \text { or }-i ;} \\
{\left[\begin{array}{rr}
-1 & 1 \\
-1 & 0
\end{array}\right] \times\left[\begin{array}{ll}
u & s u \\
0 & u
\end{array}\right], \quad s \in \mathbf{Z}, u=i \text { or }-i ;} \\
{\left[\begin{array}{rrrr}
0 & 0 & 1 & 0 \\
0 & i & 0 & 0 \\
-1 & 0 & 0 & 0 \\
0 & 0 & 0 & i
\end{array}\right]\left[\begin{array}{llll}
1 & 0 & 0 & s_{12} \\
0 & 1 & \bar{s}_{12} & s_{2} \\
0 & 0 & 1 & 0 \\
0 & 0 & 0 & 1
\end{array}\right], \quad s_{12} \in \mathbf{Z}[i], s_{2} \in \mathbf{Z} .}
\end{gathered}
$$

In this section we compute contributions from conjugacy classes in $\Gamma_{2}^{1} \cap \operatorname{Sp}(2, \mathbf{Z})$ and (1) and (2) above.

Proposition 3. Suppose

$$
M=\left[\begin{array}{ll}
a & b \\
c & d
\end{array}\right] \times\left[\begin{array}{ll}
u & 0 \\
0 & u
\end{array}\right] \in \Gamma_{2}(\mathbf{Z}[i])
$$


with $\left[\begin{array}{ll}a & b \\ c & d\end{array}\right]$ conjugate in $\mathrm{SL}_{2}(\mathbf{R})$ to $\left[\begin{array}{cc}\cos \theta & \sin \theta \\ -\sin \theta & \cos \theta\end{array}\right] \quad(\theta=\pi / 2, \pi / 3,2 \pi / 3,3 \pi / 2$, $5 \pi / 3,4 \pi / 3), u= \pm 1, \pm i, u \neq \pm 1$ if $\theta=\pi / 2$ or $3 \pi / 2)$. Then the contribution of the conjugacy class represented by $M$ to $\operatorname{dim}_{\mathbf{C}} S\left(k ; \Gamma_{2}(\mathbf{Z}[i])\right)$ is given by

$$
\begin{aligned}
N_{\{M\}}= & \frac{1}{12|G|} \frac{(u \bar{\lambda})^{k}}{(1-u \bar{\lambda})^{2}(1-\bar{u} \bar{\lambda})^{2}\left(1-\bar{\lambda}^{2}\right)} \\
& \cdot\{(k-3)(1-u \bar{\lambda})(1-\bar{u} \bar{\lambda})+2-(u+\bar{u}) \bar{\lambda}\} .
\end{aligned}
$$

Here $\lambda=e^{i \theta}$ and $|G|$ is the order of centralizer $G$ of $\left[\begin{array}{ll}a & b \\ c & d\end{array}\right]$ in $\mathrm{SL}_{2}(\mathbf{Z})$.

Proof. The centralizer of $M$ in $\Gamma_{2}(Z[i])$ contains $E_{2} \times \mathrm{SL}_{2}(\mathbf{Z})$ as a subgroup of index $|G|$. Thus we can use a fundamental domain for $E_{2} \times \mathrm{SL}_{2}(\mathbf{R})$ in our calculation of the contribution. Also it is easier to use the holomorphic images of $H_{2}, D_{2}$ : $W \in M_{2}(\mathbf{C}), E_{2}-W^{t} \bar{W}>0$, as the domain. From a similar argument on p. 98 of [5], we get $N_{\{M\}}=\pi / 12|G| \cdot I(M)$ with

$$
\begin{aligned}
I(M)= & (k-1)(k-2)^{2}(k-3) \pi^{-4}(u \bar{\lambda})^{k} \\
& \times \int_{D_{2}, w_{1}=0} \operatorname{det}\left(E-W^{\mathrm{t}} \bar{W}\right)^{k-4} \operatorname{det}\left(E-\bar{\Lambda}_{1} W \bar{\Lambda}_{2}{ }^{\mathrm{t}} \bar{W}\right)^{-k} d W, \\
\Lambda_{1}= & \operatorname{diag}[u, \lambda], \quad \Lambda_{2}=\operatorname{diag}[\bar{u}, \lambda] .
\end{aligned}
$$

Now a direct elementary calculation shows that

$$
\begin{aligned}
I(M)= & \frac{\pi^{-1}(u \bar{\lambda})^{k}}{(1-u \bar{\lambda})^{2}(1-\bar{u} \bar{\lambda})^{2}\left(1-\bar{\lambda}^{2}\right)} \\
& \cdot\{(k-3)(1-u \bar{\lambda})(1-\bar{u} \bar{\lambda})+2-(u+\bar{u}) \bar{\lambda}\} .
\end{aligned}
$$

This proves our assertion.

In the same way, we get

Proposition 4. Suppose $M \in \Gamma_{2}^{1}$ and $M$ is conjugate in $\mathrm{SU}(2,2)$ to

$$
\left[\begin{array}{rr}
\cos \theta & \sin \theta \\
-\sin \theta & \cos \theta
\end{array}\right] \times\left[\begin{array}{cc}
u & s u \\
0 & u
\end{array}\right]
$$

where $\theta$ and $u$ are as in Proposition 3, $s \in H$, a discrete subset of $\mathbf{R}-\{0\}$. For fixed $\theta$, the contribution of conjugacy classes represented by $M_{s}^{\prime}$ as $s$ ranges over $H$ is

$$
N_{\left\{M^{\prime} s\right\}}^{\prime}=\frac{1}{\left|G^{\prime}\right|} \cdot \frac{(u \bar{\lambda})^{k}}{(1-u \bar{\lambda})(1-\bar{u} \bar{\lambda})\left(1-\bar{\lambda}^{2}\right)} \lim _{\varepsilon \rightarrow 0} \sum_{s \in H}\left(\frac{1}{i s}\right)^{1+\varepsilon} .
$$

Here $G^{\prime}$ is the maximal torsion subgroup of centralizer of $M$ in $\Gamma_{2}(\mathbf{Z}[i])$, and $\left|G^{\prime}\right|$ is the order of $G^{\prime}$.

With Propositions 3,4 and the conjugacy classes of $\Gamma_{2}^{1}$ we get

Proposition 5. The contributions from conjugacy classes represented by elements in $\Gamma_{2}^{1}$, with the exclusion of those with characteristic polynomial $\left(X^{2}+1\right)(X-i)^{2}$, are 
given by the sum of the following:

1. $2^{-4} 3^{-2}(k-3) \times[1,0,-1,-1,0,1 ; 12]+2^{-3} 3^{-2} \times[0,-1,-1,0,1,1 ; 12]$,

2. $2^{-4} 3^{-2}(k-3) \times[1,0,-1,-1,0,1 ; 12]+2^{-3} 3^{-2} \times[0,-1,-1,0,1,1 ; 12]$,

3. $2^{-4} 3^{-2}(k-3) \times[-1,0,1 ; 6]+2^{-3} 3^{-2} \times[-1,1,0 ; 6]$,

4. $2^{-4} 3^{-3}(k-3) \times[1,0,-1 ; 6]+2^{-3} 3^{-4} \times[1,1,-2 ; 6]$,

5. $-2^{-6} 3^{-1} \times[1,-1 ; 4]$,

6. $2^{-2} 3^{-1} \times[-1,0,1,1,0,-1 ; 12]$,

7. $2^{-2} 3^{-1} \times[-1,0,1,1,0,-1 ; 12]$,

8. $2^{-2} 3^{-1} \times[1,0,-1 ; 6]$,

9. $2^{-2} 3^{-1} \times[-1,0,1 ; 6]+2^{-1} 3^{-3}[-1,2,-1]$.

REMARK. The sum is given by

$$
\begin{aligned}
& 2^{-2} 3^{-3}(k-2) \times[1,0,-1,-2,0,2 ; 12] \\
& \quad+2^{-3} 3^{-2} \times[-1,-2,-1,1,2,1 ; 12] \\
& \quad+17 \cdot 2^{-3} 3^{-4} \times[-1,2,-1 ; 6]-2^{-6} 3^{-1} \times[1,-1 ; 4] .
\end{aligned}
$$

4. Contribution from conjugacy classes represented by unipotent elements. An element with characteristic polynomial $(X-1)^{4}$ is conjugate in $\Gamma_{2}(\mathbf{Z}[i])$ to a $\left[S, E_{2}\right]$ with $S$ a Hermitian matrix. The total contribution of conjugacy classes of this type is given by a polynomial of degree 4 in $k$ [16]. Here we shall get a more precise result.

Proposition 5. The contribution of the identity to the dimension formula is given by

$$
2^{-6} \pi^{-4} \operatorname{vol}\left(\Gamma_{2}(\mathbf{Z}[i]) \backslash H_{2}\right)(k-1)(k-2)^{2}(k-3) .
$$

Proof. It follows from the fact that the contribution is given by

$$
2^{-6} \pi^{-4}(k-1)(k-2)^{2}(k-3) \int_{F_{2}}(\operatorname{det} Y)^{-4} d X d Y .
$$

Proposition 6. The contribution of conjugacy classes represented by $\left[S, E_{2}\right]$, with rank $S=1$, to the dimension formula is zero.

Proof. A complete set of representatives for such conjugacy classes is given by [ $\left.S, E_{2}\right], S=\operatorname{diag}[0, s], s \in \mathbf{Z}-\{0\}$. The centralizer of such elements is $\Gamma_{2}^{1}$. Hence we can use $F: Z=\operatorname{diag}[i, i y], y>0$, as a fundamental domain in our calculation; see also [5]. Note that

$$
\int_{0}^{\infty} \frac{y^{k-4} d y}{(y+i s / 2)^{k}}=\left(\frac{2}{i s}\right)^{3} \frac{1}{(k-1)(k-2)(k-3)} .
$$

Hence the total contribution is

$$
2^{-6} \pi^{-4} \operatorname{vol}\left(\Gamma_{2}^{1} \backslash \Gamma_{2, \mathbf{C}}^{1}\right)(k-2) \cdot \sum_{s \in \mathbf{Z}-\{0\}}\left(\frac{2}{i s}\right)^{3}=0 .
$$

Here $\Gamma_{2, \mathrm{C}}^{1}$ is the centralizer in $\mathrm{SU}(2,3)$ of a representative. 
Proposition 7. The contribution of conjugacy classes represented by $\left[S, E_{2}\right]$ with rank $S=2$, to the dimension formula is a constant independent of $k$.

Proof. It follows from a similar argument of Shintani [16].

Combining the propositions above, we then get

Proposition 8. The contribution from conjugacy classes represented by unipotent elements to the dimension formula is given by

$$
2^{-6} \operatorname{vol}\left(\Gamma_{2}(\mathbf{Z}[i]) \backslash H_{2}\right) \pi^{-4}(k-1)(k-2)^{2}(k-3)+\delta
$$

with $\delta$ a constant.

5. The remaining contributions and the final formula. The remaining contributions come from conjugacy classes in $\Gamma_{2}^{0}$ and those elements with characteristic polynomial $\left(X^{2}+1\right)(X-i)^{2}$. Since the set of fixed points on $H_{2}$ for any such element is a variety of dimension no more than two, the total contribution is given by $P(k)+Q(k) \times[1,-1 ; 4]$ with $P(k)$ and $Q(k)$ being polynomials of degree 2 by the Hirzebruch proportionality principle [14]. Also, these contributions can be computed directly by similar arguments as in [5 or 11].

To save space, we only calculate here those contributions from elements with characteristic polynomial $(X-1)^{2}(X+1)^{2}$. Let $M=E_{2} \times\left(-E_{2}\right), \quad G$ be the centralizer of $M$ in $\mathrm{SU}(2,2)$ and

$$
G^{\prime}=\left\{M \in G \mid M=\left[\begin{array}{ll}
a & b \\
c & d
\end{array}\right] \times\left[\begin{array}{ll}
p & q \\
r & s
\end{array}\right] \in \mathrm{SU}(2,2)\right\} .
$$

Then it is easy to see that $G^{\prime}$ is a subgroup of $G$ of index 2 .

Lemma. A fundamental domain for $\mathrm{G}^{\prime}$ on $\mathrm{H}_{2}$ is given by

$$
F_{1}: Z_{0}=\left[\begin{array}{cc}
i & t+i p \\
t-i p & i
\end{array}\right], \quad t \geqslant 0, p \geqslant 0 .
$$

The stabilizer of $Z_{0} \in F_{1}$ in $G^{\prime}$ is

$$
\left[\begin{array}{cc}
\cos \zeta & \mu^{-1} \sin \zeta \\
-\mu \sin \zeta & \cos \zeta
\end{array}\right] \times\left[\begin{array}{cc}
\cos \zeta & -\mu^{-1} \sin \zeta \\
\mu \sin \zeta & \cos \zeta
\end{array}\right], \quad \zeta \in \mathbf{R}, \mu=\sqrt{1+t^{2}+p^{2}}
$$

for $t \neq 0$ and $p \neq 0$.

Proof. See p. 115 of [5].

Proposition 9. The contribution of elements conjugate in $\Gamma_{2}(\mathbf{Z}[i])$ to $M=E_{2} \times$ $\left(-E_{2}\right)$ is given by $N_{\{M\}}=2^{-10} 3^{-2}(k-1)(k-2)$.

Proof. With the fundamental domain $F_{1}$ as constructed in Lemma 3, we then have

$$
\operatorname{det}\left\{\frac{1}{2 i}\left[Z_{0}-{ }^{\mathrm{t}} \overline{M\left(Z_{0}\right)}\right]\right\}=1+t^{2}+p^{2}
$$


Thus the contribution is (refer to [5])

$$
\begin{aligned}
N_{\{M\}} & =2^{-6} \pi^{-4}(k-1)(k-2)^{2}(k-3) \cdot \frac{\pi^{4}}{36} \cdot \int_{0}^{\infty} \int_{0}^{\infty}\left(1+t^{2}+p^{2}\right)^{-k+1} t p d t d p \\
& =2^{-10} 3^{-2}(k-1)(k-2) .
\end{aligned}
$$

Proposition 10. The contribution of conjugacy classes in $\Gamma_{2}(\mathbf{Z}[i])$, represented by $M(s)=E_{2} \times\left[\begin{array}{cc}-1 & s \\ 0 & -1\end{array}\right]$ as $s$ ranges over all nonzero integers, is given by $N^{\prime}=$ $-2^{-7} 3^{-1}(k-2)$.

Proof. The centralizer of $M(s)$ in $\mathrm{SU}(2,2)$ is a subset of $G^{\prime}$ consisting of elements of the form

$$
\left[\begin{array}{ll}
a & b \\
c & d
\end{array}\right] \times\left[\begin{array}{cc}
r & * \\
0 & r^{-1}
\end{array}\right]
$$

Thus we can use the following subset of $H_{2}$ as a fundamental domain:

$$
F_{2}: Z=\left[\begin{array}{cc}
i & z_{12}+i \tilde{z}_{12} \\
z_{12}-i \tilde{z}_{12} & i y
\end{array}\right], \quad y-y_{12}^{2}-\tilde{y}_{12}^{2}>0 .
$$

Hence

$$
\operatorname{det}\left\{\frac{1}{2 i}\left(Z-{ }^{\mathrm{t}} \overline{M(s)(Z)}\right)\right\}=\left(y-\frac{i s}{2}+x_{12}^{2}+\tilde{x}_{12}^{2}\right),
$$

and the total contribution is

$$
\begin{aligned}
N^{\prime}= & 2^{-6} \pi^{-4}(k-1)(k-2)^{2}(k-3) \cdot \frac{\pi}{12} \\
& \times \lim _{\varepsilon \rightarrow 0} \sum_{s \neq 0} \int_{F_{2}}\left(y-\frac{i s}{2}+x_{12}^{2}+\tilde{x}_{12}^{2}\right)^{-k+1}\left(y-y_{12}^{2}-\tilde{y}_{12}^{2}\right)^{k-4-\varepsilon} d Z \\
= & 2^{-6} \pi^{-4}(k-1)(k-2)^{2}(k-3) \lim _{\varepsilon \rightarrow 0} \sum_{s \neq 0}\left(\frac{1}{-i s}\right)^{1+\varepsilon} \frac{\pi^{3}}{12}(k-1)(k-2)(k-3) \\
= & -2^{-7} 3^{-1}(k-2) .
\end{aligned}
$$

Now, a case by case discussion, we get the following:

Proposition 11. The contribution from conjugacy classes in $\Gamma_{2}^{0}$ (excluding those elements with characteristic polynomial $\left.(X-1)^{4}\right)$ and those elements with characteristic polynomial $\left.\left(X^{2}+1\right)(X-i)^{2}\right)$ is given by

$$
\begin{aligned}
305 \cdot 2^{-10} 3^{-3}(k-2)^{2}-2^{-4}(k-2)+\delta_{1} \\
+\left\{276 \cdot 2^{-10} 3^{-3}(k-2)^{2}-2^{-4} 3^{-1}(k-2)+\delta_{2}\right\} \times[1,-1 ; 4] .
\end{aligned}
$$

Here $\delta_{1}$ and $\delta_{2}$ are constants. 
Combining all results obtained thus far, we now have

Proposition 12. For an even integer $k>6$, the dimension formula for the vector space of hermitian modular cusp forms is

$$
\begin{aligned}
\operatorname{dim}_{\mathbf{C}} S\left(k ; \Gamma_{2}(\mathbf{Z}[i])\right)= & 2^{-6} \pi^{-4} \operatorname{vol}\left(\Gamma_{2}(\mathbf{Z}[i]) \backslash H_{2}\right)(k-1)(k-2)^{2}(k-3) \\
& +2^{-10} 3^{-3}(k-2)^{2} \times[581,29 ; 4] \\
& -2^{-3} 3^{-3}(k-2) \times[16,9,20,13,8,5 ; 12] \\
& +5^{-2} \times[3,-2,3,-2,-2 ; 10] \\
& +4 \cdot 3^{-3} \times[-1,-2,-1,1,2,1 ; 12] \\
& +7 \cdot 2^{-1} 3^{-3} \times[-1,2,-1 ; 6] \\
& +\alpha+\beta \times[1,-1 ; 4] .
\end{aligned}
$$

Here $\alpha$ and $\beta$ are constants.

So by Proposition 12, the dimension formula will be explicit provided that we can determine $\operatorname{vol}\left(\Gamma_{2}(\mathbf{Z}[i]) \backslash H_{2}\right), \alpha$ and $\beta$.

Proposition 13. $\operatorname{vol}\left(\Gamma_{2}(\mathbf{Z}[i]) \backslash H_{2}\right)=2^{-4} 3^{-3} 5^{-1} \pi^{4}$.

Proof. Let $\delta$ be the volume. Note that the domain $G$ defined by $G: Z \in H_{2}$, $-1 / 2 \leqslant x_{1}, x_{12}, x_{12}, x_{2} \leqslant 1 / 2, \sqrt{3} / 2 \leqslant y_{1} \leqslant y_{2}, 0 \leqslant 2 y_{12}, 2 y_{12}<y_{1}$, as shown in [9], contains a fundamental domain for $\Gamma_{2}(\mathbf{Z}[i])$. Thus we have $\delta<\operatorname{vol}(G)<\pi / 18$.

On the other hand, we separate all terms in the dimension formula of Proposition 12 into two sums. Let

$$
\begin{aligned}
& Q(k) \stackrel{\doteq}{=} 2^{-6} \pi^{-4} \delta(k-1)(k-2)^{2}(k-3)+5^{-2}[3,-2,3,-2,-2 ; 10], \\
& P(k)=\text { sum of remaining terms. }
\end{aligned}
$$

It is easy to see that $P(k)=\sum_{j=0}^{2} C_{j}(k)(k-2)^{2}$ with $C_{j}(k+12)=C_{j}(k)$. For any real-valued function $f$ defined on $\mathbf{Z}$, we define

$$
\Delta f(k)=f(k+12)-f(k), \quad \Delta^{n+1} f(k)=\Delta^{n}(\Delta f(k)) .
$$

Then $\Delta^{n}(P(k)+Q(k)) \in \mathbf{Z}$ and $\Delta^{3} P(k)=0$, it follows that $\Delta^{3} Q(k) \in \mathbf{Z}$. From this fact we get a general solution for $\delta$, which is given by

$$
\delta=\left(2^{-4} 3^{-3} 5^{-1}+2^{-4} 3^{-4} \mu\right) \pi^{4}, \quad \mu \in \mathbf{Z} .
$$

By the estimate $\delta<\pi / 18$, we then have $\mu=0$ or 1 . If we assume $\mu=1$, then a direct computation shows that $\Delta^{2}(P(k)+Q(k))$ is not an integer for some $k$. Hence $\mu=0$ and the proof is completed.

It is possible to compute $\beta$ directly, but it takes a lot of calculation. Here we use the result from [9] which gives

$$
\operatorname{dim}_{\mathbf{C}} S\left(8 ; \Gamma_{2}(\mathbf{Z}[i])\right)=1, \quad \operatorname{dim}_{\mathbf{C}} S\left(10 ; \Gamma_{2}(\mathbf{Z}[i])\right)=0 .
$$

With these two conditions, we are able to determine $\alpha$ and $\beta$ explicitly as

$$
\alpha=373 \cdot 2^{-73^{-3}}+2 \cdot 5^{-2}, \quad \beta=245 \cdot 2^{-7} 3^{-3} .
$$

Consequently, we get our final formula. 
MAIN TheOREM. Suppose $k$ is an even integer greater than 6. Then

$$
\begin{aligned}
\operatorname{dim}_{\mathbf{C}} S\left(k ; \Gamma_{2}(\mathbf{Z}[i])\right)= & 2^{-10} 3^{-3} 5^{-1}(k-1)(k-2)^{2}(k-3) \\
& +2^{-10} 3^{-3}(k-2)^{2}[581,29 ; 4] \\
& -2^{-3} 3^{-3}(k-2) \times[16,9,20,13,8,5 ; 12] \\
& +2^{-6} 3^{-2}[-249,0,-57,128,423,-64 ; 12] \\
& +5^{-1}[1,0,1,0,0 ; 10] .
\end{aligned}
$$

Here $\alpha(k)=\left[a_{0}, a_{1}, \ldots, a_{m-1} ; 2 m\right]$ means $\alpha(k)=a_{j}$ if $k \equiv 2 j(\bmod 2 m)$ for $0 \leqslant j$

\begin{tabular}{|c|c|c|c|c|c|c|c|c|c|c|c|c|c|c|c|}
\hline & $k$ & & & 6 & 8 & $10 \quad 12$ & 14 & 16 & 18 & $20 \quad 22$ & 24 & 262 & \multirow[b]{3}{*}{56} & \multirow{3}{*}{\multicolumn{2}{|c|}{60}} \\
\hline \multicolumn{4}{|c|}{$\operatorname{dim}_{\mathbf{C}} S\left(k ; \Gamma_{2}(\mathbf{Z}[i])\right)$} & 0 & 1 & 0 & 0 & 3 & 0 & 1 & 10 & 21 & & & \\
\hline 30 & 32 & 34 & 36 & 38 & 40 & 42 & 44 & 46 & 48 & 50 & 52 & 54 & & & \\
\hline 4 & 23 & 8 & 31 & 12 & 42 & 18 & 57 & 28 & 73 & 39 & 93 & 53 & 119 & 73 & 148 \\
\hline 62 & 64 & 66 & 68 & 70 & 72 & 74 & 76 & 78 & 80 & 82 & 84 & 86 & & & \\
\hline 95 & 182 & 122 & 224 & 158 & 271 & 197 & 325 & 243 & 390 & 301 & 462 & 364 & & & \\
\hline 88 & 90 & 92 & 94 & 96 & 98 & 100 & 102 & 104 & 106 & 108 & 110 & & & & \\
\hline 543 & 437 & 638 & 525 & 743 & 620 & 860 & 728 & 994 & 858 & 1141 & 992 & & & & \\
\hline 112 & & & & & & & & & & & & & & & \\
\hline
\end{tabular}
$\leqslant m-1$.

With the result in the Main Theorem and the help of the computer, we get Table 1 which shows the explicit values of $\operatorname{dim}_{\mathbf{C}} S\left(k ; \Gamma_{2}(\mathbf{Z}[i])\right)$ when $6 \leqslant k \leqslant 112$.

\section{TABLE 1}

Again, with the help of the computer, we get

$$
\sum_{k=8}^{\infty} \operatorname{dim}_{\mathbf{C}} S\left(k ; \Gamma_{2}(\mathbf{Z}[i])\right) T^{k}=\frac{P(T)}{\left(1-T^{4}\right)\left(1-T^{10}\right)\left(1-T^{12}\right)^{2}\left(1-T^{16}\right)}
$$

with

$$
\begin{aligned}
P(T)= & T^{8}+2 T^{16}-T^{18}+2 T^{20}+T^{22}+2 T^{24}-T^{26}+T^{28} \\
& -2 T^{32}-T^{36}-2 T^{40}+2 T^{42}+T^{46}+T^{48}+2 T^{50}
\end{aligned}
$$

This fact can also be verified directly by calculation of residues.

REMARK. In [9] it was proved that Hermitian modular forms of degree 2 are generated by modular forms of weight $4,8,10,12,12,16$; i.e. they are generated by $\phi_{4}, \phi_{8}, \theta, \phi_{12}, \eta_{6}^{2}, \phi_{16}$ in the notation of [9, Satz 5].

\section{REFERENCES}

1. Hel Braun, Hermitian modular functions, Ann. of Math. (2) 50 (1949), 827-855.

2. , Hermitian modular functions, III, The Hermitian modular group, Ann. of Math. (2) 53 (1951), 143-180.

3. U. Christian, A reduction theory for symplectic matrices, Math. Z. 101 (1967), 213-244.

4. __ Zur Theorie der symplekischen Gruppen, Acta Arith. 24 (1973), 61-85. 
5. Minking Eie, Siegel cusp forms of degree two and three, Mem. Amer. Math. Soc., No. 304 (1984), $1-185$.

6. Contributions from conjugacy classes of regular elliptic elements in $\operatorname{Sp}(n, Z)$ to the dimension formula, Trans. Amer. Math. Soc. 285 (1984), 403-410.

7. Contributions from conjugacy classes of regular elliptic elements in hermitian modular groups to the dimension formula of hermitian modular cusp forms, manuscript (1985).

8. Minking Eie and Chung-Yuan Lin, Fixed points and conjugacy classes of regular elliptic elements in $\mathrm{Sp}(3, Z)$, Trans. Amer. Math. Soc. 289 (1985), 485-496.

9. E. Freitag, Modulformen zweiten Grades zum rationalen und Gaußschen Zahlkörper, S.-B. Heidelberger Akad. Wiss. Math.-Natur. Kl., 1967, pp. 3-49.

10. R. Godement, Fonctions automorphes, Séminaire, 1957/1958.

11. K. Hashimoto, The dimension of spaces of cusp forms on Siegel upper half plane of degree two (I), J. Fac. Sci. Univ. Tokyo Sect. IA Math. 30 (1983), 438-488.

12. K. Hashimoto and T. Ibukiyama, On class numbers of positive binary quaternion hermitian forms (II), (III), J. Fac. Sci. Univ. Tokyo Sect. IA Math. 28 (1981), 695-699; 30, (1983), 393-400.

13. R. P. Langlands, Dimension of spaces of automorphic forms, Amer. J. Math. 85 (1963), 99-125.

14. D. Mumford, Hirzebruch's proportionality theorem in the noncompact case, Invent. Math. 42 (1977), 239-272.

15. Y. Morita, An explicit formula for the dimension of spaces of Siegel modular forms of degree two, J. Fac. Sci. Univ. Tokyo Sect. IA Math. 21 (1974), 167-248.

16. T. Shintani, On zetafunctions associated with the vector space of quadratic forms, J. Fac. Sci. Univ. Tokyo 22 (1975), 25-65.

Institute of Mathematics, Academia Sinica, Nankang, Taipei, Taiwan

Mathematisches Institut der Universität GOttingen, GOtTingen, Federal Republic of GERMANY (Current address) 